Breakup of Liquid Filaments

Alfonso A. Castrejón-Pita, J. R. Castrejón-Pita, and I. M. Hutchings

Phys. Rev. Lett. 108, 074506 (2012) 


\title{
The breakup of liquid filaments
}

\author{
A.A. Castrejón-Pita, J.R. Castrejón-Pita, and I.M. Hutchings \\ Department of Engineering, University of Cambridge, \\ 17 Charles Babbage Road, Cambridge, CB3 OFS, U.K.
}

\begin{abstract}
Whether a thin filament of liquid separates into two or more droplets or eventually condenses lengthwise to form a single larger drop depends on the liquid's density, viscosity and surface tension and on the initial dimensions of the filament. Surface tension drives two competing processes, pinching-off and shortening, and the relative timescales of these, controlled by the balance between capillary and viscous forces, determine the final outcome. Here we provide experimental evidence for the conditions under which a liquid filament will break up into drops, in terms of a wide range of two dimensionless quantities: the aspect ratio of the filament and the Ohnesorge number. Long filaments which do not break up into multiple droplets demand a high liquid viscosity or a small filament aspect ratio.
\end{abstract}

PACS numbers: 47.55.D-, 47.55.db and 47.80.Jk

The difference in behaviour between thin streams of various liquids is familiar in the formation of droplets from a thin water stream trickling from a tap, and in the quite different behaviour of the more viscous threads of honey or syrup flowing from a spoon [1]. The breakup of liquid jets is important in several practical contexts, including the dispersion of liquid drugs into respirable droplets, microfluidics, crop- and paint-spraying, and ink-jet printing [2-4]. There are also biological systems in which either long filaments remain intact, or many droplets are formed $[5,6]$. In some cases, such as the water jets generated by the archerfish [7], intermediate behaviour is seen and an initially continuous jet breaks up eventually into drops. A geological example of liquid threads that do not break up is provided by the very fine filaments of glassy lava, widely known as Pele's hair, produced by certain volcanic eruptions $[8,9]$.

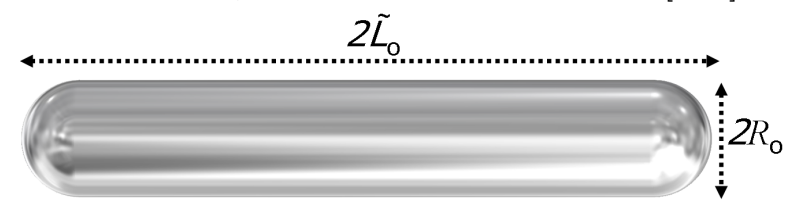

FIG. 1: (Colour online) Geometry of a free cylindrical liquid filament with spherical end caps as assumed in the models of Schulkes [10] and Notz and Basaran [11].

Theoretical models for the collapse of a thin filament of a Newtonian liquid (i.e. in which viscosity is independent of shear rate) of finite length, as shown in Fig. 1, have been proposed by Schulkes [10] and Notz and Basaran [11], but experimental evidence for their predictions has so far been very limited. This is largely because most experimental techniques can cover only a small range of fluid properties and filament length. Furthermore, it is not easy in practice to generate the free cylindrical filaments that these models assume, and most studies have therefore explored the quite different geometries of a continuous jet or of liquid dripping from a tube [1]. We have used an apparatus which was originally designed to replicate jet and droplet formation in an ink-jet printer, but on a larger scale [12], to produce free cylindrical filaments of Newtonian liquids and explore their collapse behaviour over a wide range of aspect ratios and liquid properties. The results provide experimental confirmation of important aspects of theoretical models and also demonstrate some limitations.

The relative importance of surface tension and viscosity in the behaviour of a liquid filament is expressed through the Ohnesorge number $\mathrm{Oh}=\frac{\mu}{\sqrt{\rho \sigma R_{0}}}$, where $\mu$, $\rho$ and $\sigma$ are the viscosity, density and surface tension of the liquid respectively and $R_{o}$ is the initial radius of the filament. The initial geometry of a filament of length $2 \tilde{L}_{o}$ can be described by the aspect ratio $L_{o}=\tilde{L}_{o} / R_{o}$.

The models of Schulkes and Notz and Basaran used combined Lagrangian-Eulerian methods (Galerkin finite element model, G/FEM) to solve the dynamics of incompressible Newtonian liquid filaments surrounded by an inert ambient fluid, [10] and [11]. Due to computational limitations, Schulkes' simulations could not follow the dynamics to breakup and therefore the results are mostly based on neck contraction rates. Despite its limitations, this pioneering study provided the first insights into the existence of a critical Oh number $\left(\mathrm{Oh}_{c}\right)$ separating regimes in which a filament would breakup or not. Notz and Basaran performed a more detailed and extensive exploration of the parametric space to confirm the previous hypotheses that the initial filament aspect ratio $L_{o}$ is a crucial parameter that, together with the $\mathrm{Oh}$ number, determines the fate of liquid filaments. Schulkes [10] predicted that a filament for which $\mathrm{Oh} \geq \mathrm{O}(1)$ will contract into a droplet regardless of its initial aspect ratio $L_{o}$, while a sufficiently long filament with $\mathrm{Oh} \leq$ $\mathrm{O}(0.01)$ will form bulges separated by thinner filaments and ultimately break up into separate droplets. Moreover, Schulkes predicted that $\mathrm{Oh}_{c}$ lies within the range of $0.005<O h<0.01$ for $8 \leq L_{o} \leq 15$. Notz and Basaran [11] went further, by concluding that for low Ohnesorge numbers, $\mathrm{Oh} \leq \mathrm{O}(0.1)$, the final breakup is governed by both a critical value $\left(\mathrm{Oh}_{c}\right)$ and a critical initial aspect ratio $\left(L_{o, c}\right)$. A filament with $L_{o}>L_{o, c}$ and $\mathrm{Oh}>\mathrm{Oh}_{c}$ will break up, whereas a filament with $L_{o}<L_{o, c}$ and $\mathrm{Oh}$ $<\mathrm{Oh}_{c}$ will not. They established explicit values for the critical Ohnesorge number and aspect ratio by computa- 
tional modelling but experimental data is not available for the $\left(\mathrm{L}_{o}, \mathrm{Oh}\right)$ parameter space. Previous studies in the context of ink-jet printing have provided only very limited experimental evidence [13, 14], and in any case typical ink-jet print heads, which can be used only with fluids with a narrow range of viscosity (typically $5-30$ $\mathrm{mPa} \mathrm{s}$ ), are restricted to the range $\sim 0.1<\mathrm{Oh}<\sim 1$. In contrast, here we have explored the behaviour over the range $0.003<\mathrm{Oh}<10$, for filaments with $1<L_{o}<70$.

We used a liquid jet generator modified from a design described elsewhere $[12,14]$ and driven by an electrical waveform to produce jets of water-glycerol mixtures which were imaged with a high-speed camera. Different types of drive waveform were required to produce free symmetrical filaments for different regimes of Oh. In all cases, the filament was formed as a secondary product of jetting, behind a primary drop or sequence of drops.

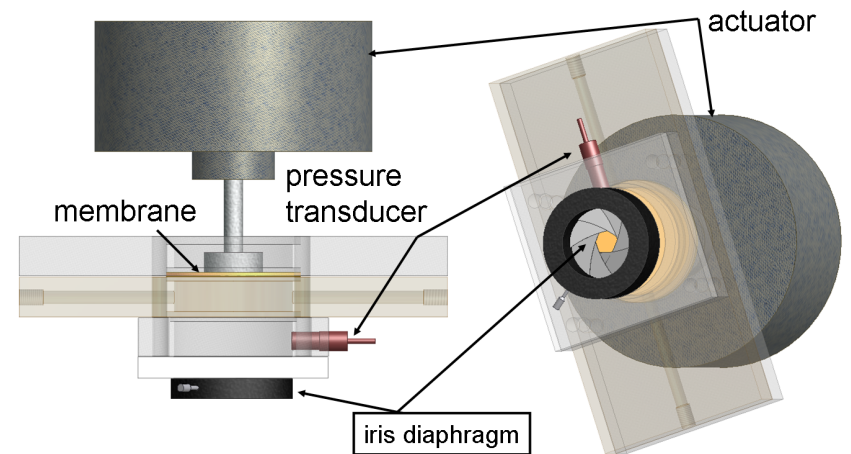

FIG. 2: (Colour online) Apparatus with a nozzle of variable diameter used to generate liquid jets.

The jet generator (schematically shown in Fig. 2) consists of a cylindrical chamber with an outlet nozzle in its lower side and closed at the top by a flexible rubber membrane which is mechanically coupled to an electromagnetic actuator (V201, LDS Instruments, UK) driven by electrical pulses from a waveform generator under computer control. The pressure history within the chamber is monitored by a transducer mounted in its side. In this work we used a variable iris diaphragm as the nozzle which could be continually varied from 0.2 to $5 \mathrm{~mm}$ in diameter to allow us to achieve a wide range of $\mathrm{Oh}$. We used mixtures of water and glycerol (including pure water and pure glycerol) with the following values of viscosity, measured at 22 Celsius: 1, 2, 5, 6, 20, 30, 50, 100, 200, 430 and $1400 \mathrm{mPa}$ s. The surface tensions lay between $72 \mathrm{mN} \mathrm{m}^{-1}$ (for pure water) and $63 \mathrm{mN} \mathrm{m}^{-1}$ (for pure glycerol). Jet ejection speeds were between 0.5 and $2.4 \mathrm{~m} \mathrm{~s}^{-1}$. We recorded images of the jet after it had emerged from the nozzle with a high-speed camera (Phantom V310, Vision Research, USA) in shadowgraph mode at a frame rate of 10,000 frames per second with an exposure time of $5 \mu \mathrm{s}$. The region of interest was illuminated from behind with a $500 \mathrm{~W}$ tungsten filament lamp through a $30 \times 30 \mathrm{~cm}^{2}$ optical diffuser. The camera was used with two different lenses to cover different fields of view: a Nikkor $18-55 \mathrm{~mm}$ for $15 \times 15 \mathrm{~cm}^{2}$, and a macro Tamron SP AF90 for $5 \times 5 \mathrm{~cm}^{2}$.
In drop-on-demand ink-jet printing, the speed of the jet is mainly controlled by the amplitude of the first voltage peak, and its diameter by the duration of this first pulse $[13,15]$. Detachment of the jet from the nozzle and the characteristics of the resulting liquid filament are, in contrast, governed by later peaks in the driving waveform. At low Ohnesorge number surface tension
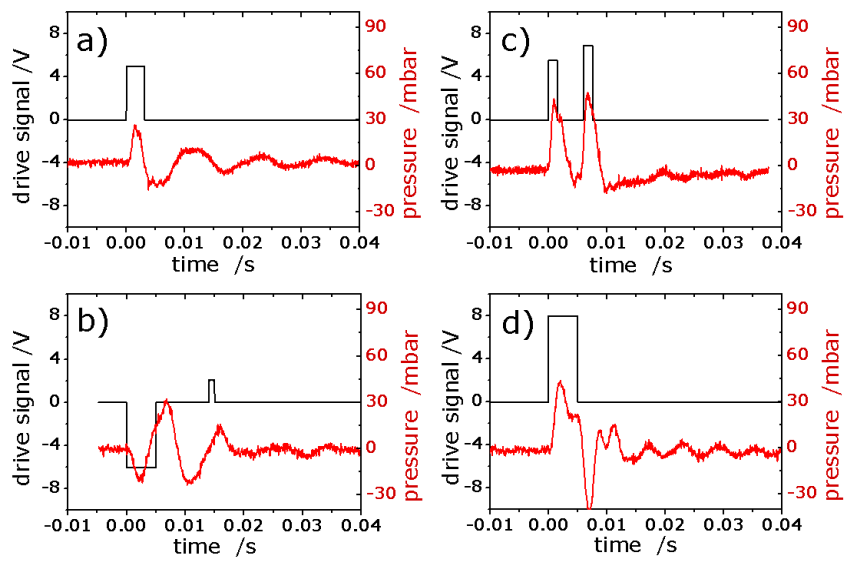

FIG. 3: (Colour online) Examples of drive voltage waveforms (upper traces) and resulting pressure waveforms (lower traces) used to produce symmetrical filaments for: (a) $\mathrm{Oh}=0.01$ and $L_{o}=2.9$; (b) $\mathrm{Oh}=0.04$ and $L_{o}=9.0$; (c) $\mathrm{Oh}=0.18$ and $\mathrm{L}_{o}$ $=29.2 ;(\mathrm{d}) \mathrm{Oh}=1.01$ and $L_{o}=59.0$.

dominates the behaviour of the liquid [16], and in the ejection of a jet from a nozzle this results on the formation of a large droplet followed by an attached filament. When the filament detaches from the nozzle its free end rapidly condenses into a smaller tail droplet. The filament then breaks up into separate droplets, usually preceded by pinching-off at the two ends, close to the two end droplets. In order to form a symmetrical cylindrical filament under these conditions, we adjusted the duration of the single-pulse driving waveform to ensure that the two ends of the central filament pinched off simultaneously, giving a free filament preceded and followed by separate drops. Figure 3(a) shows a typical drive waveform, together with the associated pressure pulse within the liquid chamber. At high Ohnesorge number, in contrast, viscous forces dominate and a different approach is needed. In this case, very long filaments (up to $150 \mathrm{~mm}$ long) were ejected by the use of long drive pulses (Fig. 3d), and their behaviour was followed until they broke up into a series of shorter filaments. We then analyzed the behaviour of these filaments.

At intermediate values of $\mathrm{Oh}$, both surface tension and viscosity play important roles and we used two different methods to create symmetrical filaments. The first was similar to that used at high Oh, and involved the production of long liquid filaments. However, as the pinching-off process occurs more rapidly, we used a pull and push waveform (Fig. 3b) to form the liquid filament more quickly: the second pulse ensured that the filament became detached from the nozzle. The second method used two positive pulses (Fig. 3c) to produce two 

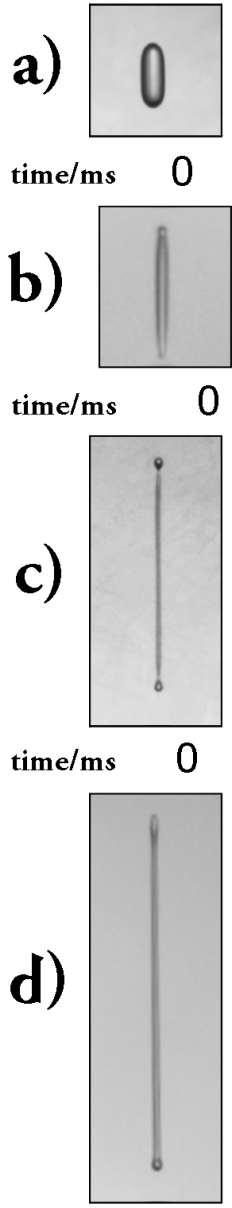

time/ms

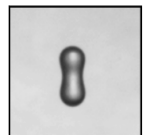

1.3

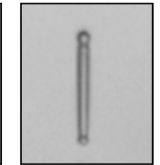

0.7

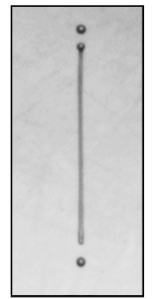

1.0

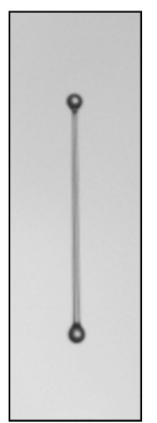

4.0

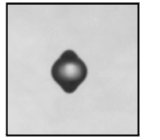

3.7

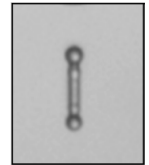

1.0

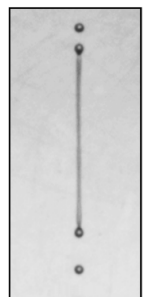

2.0

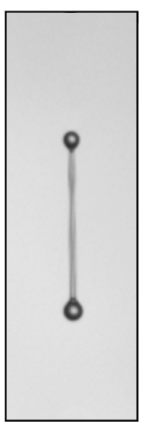

6.3

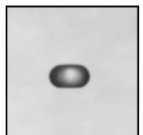

4.3

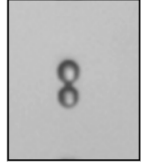

1.3

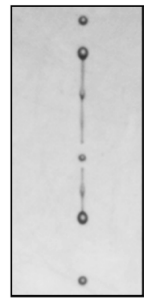

4.0

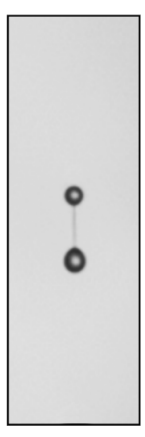

10.0

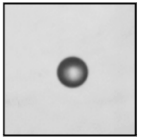

30.0
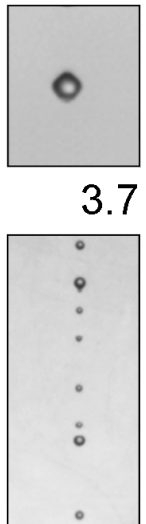

5.0

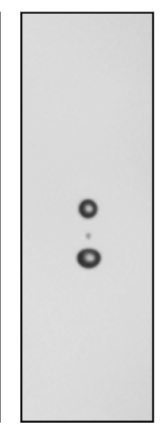

10.7

FIG. 4: Images of filaments generated by the drive waveforms shown in Figure 3, together with their shapes after the times shown, for the following liquid viscosities and filament halflengths: (a) $\mu=2 \mathrm{mPa}$ s and $R_{o}=0.95 \mathrm{~mm}$; (b) $\mu=5 \mathrm{mPa} \mathrm{s}$ and $R_{o}=0.19 \mathrm{~mm}$; (c) $\mu=20 \mathrm{mPa} \mathrm{s}$ and $R_{o}=0.22 \mathrm{~mm}$ (d) $\mu=100 \mathrm{mPa}$ s and $R_{o}=0.13 \mathrm{~mm}$.

large droplets separated by a continuous filament, which, with the correct delay between the two pulses, then became detached at both its ends simultaneously. In all our experiments the movement and development of the filaments was followed by analyzing the video recording, to ensure that they collapsed symmetrically and to establish whether they condensed axially into a single drop or broke up into two or more separate drops. Examples where collapse was not symmetrical, for example in which the initial conditions led to significant differences in the sizes of the drops forming at the ends of the filament, were discarded. Careful experimentation, involving fine tuning of the drive waveforms, was needed to produce filaments which contracted symmetrically, and to explore a wide range of values of $\mathrm{Oh}$ and $\mathrm{L}_{o}$.

The behaviour of a liquid filament moving freely in air would be expected to be influenced by the presence of the air, but this is ignored in the models under consideration. We can show that its influence was also negligible in our experiments. Viscous drag from

the surrounding air would be expected to become significant only for filaments with a radius smaller than $\sim a_{0} \mu_{\text {air }} \mathrm{Oh}^{2} / \mu=\mu \mu_{\text {air }} / \rho$ We where $\mu_{\text {air }}$ is the viscosity of air, We is the Weber number defined as $\mathrm{We}=\rho v^{2} R_{0} / \sigma$ and $v$ the speed of the filament $[16,17]$. This critical radius is $<1 \mu \mathrm{m}$ for all the liquids studied, and thus below the resolution of the imaging system we used. Inertial influences from the air would become significant when the gas Weber number [18], defined by $\mathrm{We}_{g}=\rho_{\text {air }} / \rho \mathrm{We}$ (where $\rho_{\text {air }}$ is the density of air), is greater than 0.2 . In our work $\mathrm{We}_{g}$ lay between 0.018 and 0.003 , so that aerodynamic influences from this source would also have been negligible. Higher ejection speeds would involve other type of breakup mechanisms induced by aerodynamic and viscous effects not considered in the numerical models being compared here [10, 11].

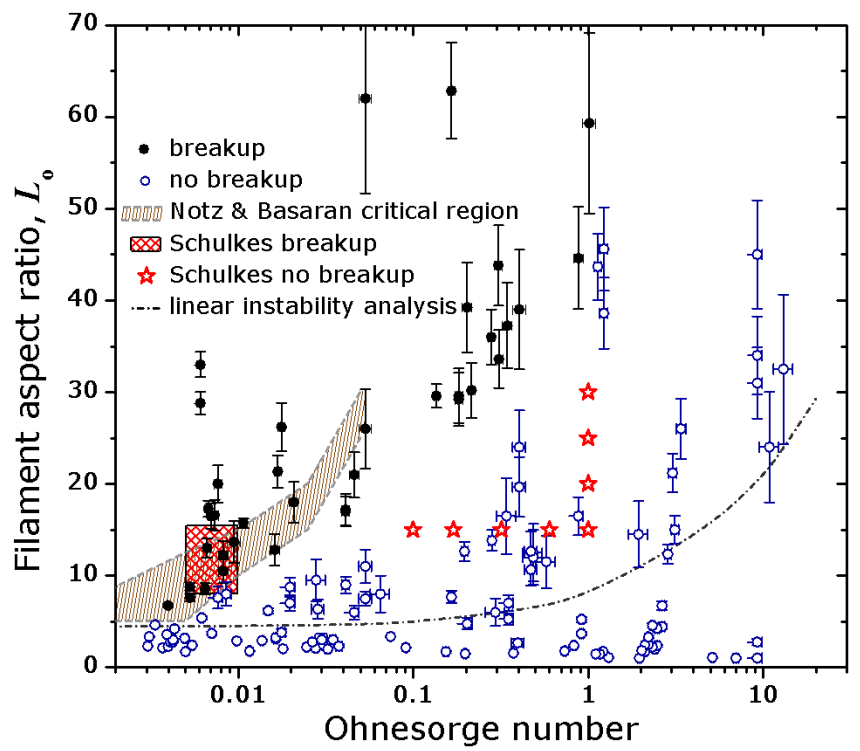

FIG. 5: (Colour online) Results plotted in terms of $\mathrm{Oh}$ and $L_{o}$. Solid symbols represent cases where the filament broke up into two or more droplets and open symbols where the filament contracted into a single droplet. The error bars represent uncertainty associated with the measurements of filament length and diameter. The shaded area shows the predictions of the model of Notz and Basaran [11]. The hatched region represent the range of critical $\mathrm{Oh}$ predicted by Schulkes [10]. Stars represent the points explored by Schulkes in which breakup does not occur. The broken line corresponds to the prediction of the linear instability analysis.

Figure 4 shows examples of typical liquid filaments with a range of different aspect ratios produced in this work by the drive waveforms shown in Fig. 3, in each case with a series of images showing their subsequent behaviour. The shortest filaments (e.g. Fig. 4a) always condensed into single drops for all values of $\mathrm{Oh}$, while for the highest values of $\mathrm{Oh}$ the filaments never broke up, whatever their aspect ratio. The fates of all the filaments are plotted in Fig. 5. Each point represents the results of a large number (up to 40) of experimental observations. Within this parameter space, there is a clear division between filaments which broke up into two or 
more droplets and those which collapsed to form a single droplet.

Within the range of Oh explored here, the results show that there is a tendency towards a critical initial aspect ratio of $6 \pm 1$ below which a liquid filament will not break up, irrespective of the value of Oh. In addition, no filament breakup was observed for $\mathrm{Oh}>1$, regardless of its initial aspect ratio. The results obtained in a more limited regime by Schulkes together with the existence of a critical value of Oh for break-up as predicted in [10] are in full agreement with our work. The predictions by Notz and Basaran [11] of the critical aspect ratio for breakup for $10^{-3}<\mathrm{Oh}<5 \times 10^{-2}$ (shown by the shaded area in Fig. 5) also agree quite well with our observations for the lowest values of $\mathrm{Oh}$, but underestimate the tendency of the filaments to break up as Oh tends towards 0.05. However, it should be noted that the shaded area is based on only five numerically-generated points.

As shown in Fig. 5, the breakup transition predicted by Notz and Basaran differs from our observations in the range $0.7<\mathrm{Oh}<0.05$. This discrepancy may be attributed to computational factors and to differences between the theoretical model and the conditions in the experiments. Notz and Basaran noted that in their G/FEM simulations, filament ratios exceeding $L_{0} \geq 15$ required more axial mesh elements and substantially longer computation times that may compromise the accuracy of the final result. In practice, there are also factors that differentiate these experiments from the simple conditions assumed by the models. Surface effects (e.g. contamination, surface vibration, filaments not at initial rest, temperature driven effects, etc.) will affect the dynamics of the liquid surface and ultimately the breakup of real filaments, [19]. In the experiments, these factors will have a different effect in different regimes. Internal dynamics are unlikely to be important for highly viscous fluids since they are quickly damped by viscous forces: the time scale over which the filament contracts is large compared with the typical time scale on which initial disturbances will attenuate, [11]. In contrast, for low Oh, the surface pinch off occurs fast and disturbances will have not enough time to grow. Surface defects will be more evident for long and thin filaments which is precisely where the predicted transition seems to be underestimated. In addition to these problems, the experimental conditions of the imaging setup were demanding, as very large fields of view and high framing speeds were needed to follow long moving filaments from initial jetting to the point of breakup. Consequently, the symmetrical shape of the filaments (or any initial surface perturbation) could only be assured down to the resolution of the camera system; the image resolution here ranged from 40 to 16 pixels $/ \mathrm{mm}$ for the longest ligaments.

Linear stability analysis offers an alternative approach to describe the pinching off of a liquid thread [2], [13] and [20]. This method assumes that the breakup is caused by the fastest growing disturbance; if the disturbance has the longest wavelength $\left(\lambda_{\max }\right)$ that fits in the finite length of the thread $\left(2 \tilde{L}_{o}\right)$ then the filament will break up (Eq. 2 in [13]). Although this method has provided good approximations for the breakup times of droplets, it does not predict the dynamics of contracting satellites or ligaments that are formed once the thread separates from both the fluid and the nozzle, as is the case in this work, [13] [20].

Our results significantly extend the range of filament length and Ohnesorge number over which filament breakup has been explored, and provide experimental validation for the main conclusions of the earlier modeling work. It is now possible to predict whether a filament will break up or not. For a Newtonian liquid, long liquid filaments which do not break up into multiple droplets can only be produced when the Ohnesorge number is large enough $(\mathrm{Oh}>1)$, which in turn demands a high viscosity or a small filament aspect ratio.

This project was supported by the UK Engineering and Physical Sciences Research Council and industrial partners in the GlassJet and I4T projects (Grant numbers $\mathrm{EP} / \mathrm{G} 029458 / 1$ and EP/H018913/1).
[1] J. Eggers and E. Villermaux, Rep. Prog. Phys. 71 0366601 (2008).

[2] J. Eggers, Rev. Mod. Physics 69865 (1997).

[3] S. Limpanuphap and B. Derby, J. Mater. Sci. Mater. Medicine 121163 (2002).

[4] O.A. Basaran, AIChE Journal 481842 (2002).

[5] T.D. Sutherland, J. H. Young, S. Weisman, C. Y. Hayashi and D.J. Merritt, Annu. Rev. Entomol. 55171 (2010).

[6] T. Eisner and D. J. Aneshansley, Proc Natl Acad Sci USA 9669705 (1999).

[7] T. Schlegel, C. J. Schmid and S. Schuster, Current Biology 16 R836 (2006).

[8] D. Shimozuru, Bull Volanol. 56217 (1994).

[9] S. Moune, F. Faure, P.J. Gauthier, K. W. W. Sims, J. Volcanol. Geoth. Res. 164244 (2007)

[10] R.M.S.M. Schulkes, J. Fluid. Mech. 309277 (1996).

[11] P.K. Notz and O.A. Basaran, J. Fluid Mech. 512223 (2004).
[12] J.R. Castrejón-Pita, G.D. Martin, S.D. Hoath, and I.M. Hutchings, Rev. Sci. Instrum. 79075108 (2008).

[13] H. Dong, W.W. Carr and J.F. Morris, Phys. Fluids 18 $072102(2006)$.

[14] J.R. Castrejón-Pita, N.F. Morrison, O.G. Harlen, G.D. Martin and I. M. Hutchings, Phys. Rev. E 83036306 (2011).

[15] A.U. Chen and O.A. Basaran, Phys. Fluids 14 L1 (2002).

[16] A.U. Chen, P.K. Notz and O.A. Basaran, Phys. Rev. Lett. 88174501 (2002).

[17] J.R. Lister and H.A. Stone, Phys. Fluids 102758 (1998).

[18] W. Hoeve, et.al., Phys. Fluids 22122003 (2010).

[19] M.A. Herrada, J.M. Montanero and J.M. Vega, Phys. Fluids 23082102 (2011).

[20] D. Henderson, H. Segur, L.B. Smolka, and M. Wadati, Phys. Fluids 12550 (2000). 